The line on the graph is the fitted curve of a simple natural logarithm equation. Because the correlation coefficient is high it is possible to predict that a successful bond will be achieved without pressure at temperatures above about $430^{\circ} \mathrm{C}$. Certainly it is the author's experience that heattreatment of a bundle of pure gold wires for one hour at $500^{\circ} \mathrm{C}$ resulted in a single rod and some hindsight into gold diffusion bonding!

\section{REFERENCES}

1 P. J. Martin, Gold Bull., 1986, 19(4), 102-116

2 D.L. Ornellas and E. Catalano, Rev. Sci. Instr., 1974, 45(7), 955

3 R.L. Williams and J.B. Tyra, Hughes Aircraft Company, WO Patent 94/17551

\title{
Low Resistance Gold Contacts for Gallium Nitride
}

\author{
Peter Boswell \\ Bricad Associates sarl, route de Genève, 7, CH-1291 Commugny, Switzerland
}

\begin{abstract}
Gold is playing an important role in the development of reliable contacts with a very low contact resistance to gallium nitride.
\end{abstract}

Owing to its chemical inertness, gold is often the ideal contact material for semiconductor devices. However, new generations of semiconductors are continually entering mainstream applications, so device manufacturers must tackle fundamental challenges when continuing to use gold in novel types of contacts. The identification of a viable contact for gallium nitride demonstrates that gold's versatility often lies at the heart of modern contact systems based on sophisticated physical concepts. The system comprises a series of thin films deposited successively on the gallium nitride where the final, relatively thick $(50 \mathrm{~nm})$ film of gold provides the low-resistance connection to the exterior.

Gallium nitride has emerged mainly because the capacities of optical storage media used in domestic audio and video equipment and in computer storage devices increase remorselessly in the quest for higher performance. Solid-state lasers working in the blue to ultraviolet are necessary, and materials based on the II-VI and III-V elements race to become the dominant system. The ZnSe II-VI compound has received the most attention, highlighted by the announcement in 1994 of lasers capable of operating for over 100 hours. However, the GaN III-V compound with superior electronic and physical properties is the material of choice. For instance, the large energy difference for electrons sitting in the conduction and valence bands leads to a small leakage current and a large breakdown voltage, important properties for devices operating at high temperatures and at high frequencies. Unfortunately, producing defect-free $\mathrm{GaN}$ crystals is extremely difficult so there is no commercial source of $\mathrm{GaN}$ substrates for growing the layer structures used in solid-state lasers.

Meanwhile, GaN grown on sapphire substrates is now the material of choice for manufacturing the lasers' cousins, namely light emitting diodes (LEDs) that operate in the blue. So the installed production capacity for high-brightness GaN LEDs has leapt from almost zero a few years ago to over 10 million units per month worldwide. This has paved the way for using GaN LEDs in ubiquitous applications such as traffic lights. The potential for high-temperature and highfrequency applications and for short-wavelength detectors persists, with overall success ultimately depending on the future development of bulk materials and layer growth processes, and on substrate technologies in general. 


\section{LOW-RESISTANCE CONTACTS}

In a semiconductor laser or LED, light is emitted when electrons, injected into the conduction band, fall into the lower energy valence band. So a high overall efficiency requires a low electrical resistance for the contact between current leads and the semiconductor. Developing stable and reliable contacts with a very low contact resistance to $\mathrm{GaN}$ is therefore a crucial issue.

If the difference between a semiconductor's valence and conduction band energies is not too large one can generally find a metal with a low enough work function which allows electrons to flow easily from the metal into the semiconductor. However, GaN's band gap energy of 3.42 electron volts is so large that a suitable metal with a small work function does not exist. Consequently, thermionic emission from the metal cannot be used as the basis for low-resistance contacts.

For an unreacted metal with a relatively large work function, the contact resistance is determined by the Schottky barrier height. Owing to the ionic nature of $\mathrm{GaN}$, it was predicted by Forest and Moustakas in 1993 that the barrier height is given by the difference between the contact metal's work function and the electron affinity in the semiconductor. Confirmation that the work function determines the contact resistance of unreacted pure metal contacts on positively doped $\mathrm{GaN}$ came three years later (1).

However, the contact resistance for platinum, which has the largest work function from among the commonly available precious metals, remained $100-$ times too large for lasers. The sought-for reduction in the contact resistance (to the $10^{-4} \mathrm{ohm} \mathrm{cm}^{2}$ ) level can possibly be achieved by reducing the barrier height - either by forming a thin intermediate semiconductor layer or by adjusting the semiconductor side of the contact ( $\mathrm{eg}$, by heavily doping the $\mathrm{GaN}$ surface layer).

\section{ELECTRON TUNNELLING}

If the energy of the GaN's conduction band near the metal-GaN interface is decreased sufficiently by doping (a process called 'band bending'), the tunnelling of carriers through a thin contact layer becomes the primary transport mechanism. Showing that an extremely low contact resistance can been achieved using this approach came quite by chance when Lin et al (2) used a thin $\mathrm{Ti}$ adhesion layer sandwiched between n-type $\mathrm{GaN}$ and an $\mathrm{Al}$ contact layer to obtain a well-bonded contact. The specific resistance they measured was only $8 \times 10^{-6} \mathrm{ohm} \mathrm{cm}^{2}$.

The $\mathrm{Ti} / \mathrm{Al}$ contact layer lost its good conductivity during thermal annealing so a second composite layer had to be evaporated onto the Al layer after the thermal treatment. This represented a supplementary manufacturing step so Fan et al (3) investigated what could be achieved by tailoring both the process used to clean the $\mathrm{GaN}$ surface and the structure of the multilayer contact. A specific resistance of $8.9 \times 10^{-8}$ ohm $\mathrm{cm}^{2}$ was obtained by depositing a Ti/Al/Ni/Au $(15 \mathrm{~nm} / 220 \mathrm{~nm} / 40 \mathrm{~nm} / 50 \mathrm{~nm})$ composite multilayer after reactive ion etching to clean the n-type $\mathrm{GaN}$ surface. The multilayer retained its low resistivity after thermal annealing, and the etching process and the formation of $\mathrm{TiN}$ at the interface introduced defects that reduced the barrier height for electron tunnelling.

Another approach for enhancing tunnelling relies on direct doping of the $\mathrm{GaN}$ surface layer. However, conventional doping techniques, based on the diffusion of alloyed ingredients from the deposited metal layer into the $\mathrm{GaN}$ to reduce the energy levels, do not work because reaction layers form during the high-temperature thermal treatments. Burm et al (4) have shown that a specific contact resistance of only $3.6 \times 10^{-8} \mathrm{ohm} \mathrm{cm}^{2}$ can be realized by implanting silicon in the n-type $\mathrm{GaN}$ before depositing a $3 \mathrm{~nm} \mathrm{Ti}$ adhesion layer and a thick (300nm) Au contact layer. Thermal annealing to activate the dopants may cause complications and implantation represents an additional process step, so this particular approach may not be commercially viable.

The work confirms nonetheless the importance of recent developments in gold-based composite layers for low resistance contacts on highly doped GaN. As Fan et al conclude: "we confidently state that extremely low ohmic contact resistance is now possible on $\mathrm{GaN}$ which can pave the way for the exploitation of this material for high power/high efficiency amplifiers".

\section{REFERENCES}

1 H. Ishikawa, S. Kobayashi, Y. Kiode, S. Yamasaki, S. Nagai, J. Umezaki, M. Kioke and M. Murakami, J. Appl. Phys, 1997, 81, 1315

2 M.E. Lin, Z.Ma, F.Y. Huang, Z.F. Fan, L.H. Allan and H.Morkoç, Appl. Phys. Lett, $1994,64,1003$

3 Z. Fan, S. Noor Mohammad, W. Kim, O. Aktas, A.E. Botcharev and H. Morkoç, Appl. Phys. Lett. 1996, 68, 1672

4 J. Burm, K. Chu, WA. Davis, W.J. Schaff and L.F. Eastman, Appl. Phys. Lett., 1997, 70,464 